\title{
VariantClassifier: A hierarchical variant classifier for annotated genomes
}

\author{
Kelvin Li* and Timothy B Stockwell
}

\begin{abstract}
Background: High-throughput DNA sequencing has produced a large number of closed and well annotated genomes. As the focus from whole genome sequencing and assembly moves towards resequencing, variant data is becoming more accessible and large quantities of polymorphisms are being detected. An easy-to-use tool for quickly assessing the potential importance of these discovered variants becomes ever important.
\end{abstract}

Findings: Written in Perl, the VariantClassifier receives a list of polymorphisms and genome annotation, and generates a hierarchically-structured classification for each variant. Depending on the available annotation, the VariantClassifier may assign each polymorphism to a large variety of feature types, such as intergenic or genic; upstream promoter region, intronic region, exonic region or downstream transcript region; 5' splice site or 3' splice site; 5' untranslated region (UTR), 3' UTR or coding sequence (CDS); impacted protein domain; substitution, insertion or deletion; synonymous or non-synonymous; conserved or unconserved; and frameshift or amino acid insertion or deletion (indel). If applicable, the truncated or altered protein sequence is also predicted. For organisms with annotation maintained at Ensembl, a software application for downloading the necessary annotation is also provided, although the classifier will function with properly formatted annotation provided through alternative means.

Conclusions: We have utilized the VariantClassifier for several projects since its implementation to quickly assess hundreds of thousands of variations on several genomes and have received requests to make the tool publically available. The project website can be found at: http://www.jcvi.org/cms/research/projects/variantclassifier.

\section{Findings}

The prevalence and increasing ubiquity of genome resequencing has greatly increased due to the emergence of lower cost deep sequencing technologies, such as Roche 454 [1] or Illumina Solexa [2], when compared to traditional Sanger sequencing. This has led to an enormous growth of variant data on well-characterized and annotated genomes. To manually filter through all the collected variant information is a daunting and error prone task, especially given the variety of information that may influence the assessment of a variant's importance. Winnowing through the possible information that can be derived from annotation is naturally a hierarchical process. For example, a single nucleotide polymorphism (SNP) in an intergenic region may be less interesting than one found in a gene, and a SNP found in an intron may be

* Correspondence: kli@jcvi.org

1 The J. Craig Venter Institute, 9704 Medical Center Drive, Rockville, MD 20850, USA

Full list of author information is available at the end of the article less interesting than one found in an exon. However, the intron/exon classification is irrelevant if the intergenic classification is already made.

Some variation annotation can be found at dbSNP [3] and Ensembl [4], after a variant has been submitted for publication, but no stand-alone or free tools currently exist for an investigator to quickly assess identified variants. We have developed VariantClassifier, an easy-to-use software tool that utilizes user-supplied genome annotation to classify variants hierarchically. When results have been loaded into a spreadsheet application, identified variants can be sorted by their classification, helping to prioritize the subsets of variants investigators may be interested in focusing on.

\section{Inputs}

There are 3 inputs into the VariantClassifier. See Figure 1 for the relationship between the inputs and the organism's genome. 


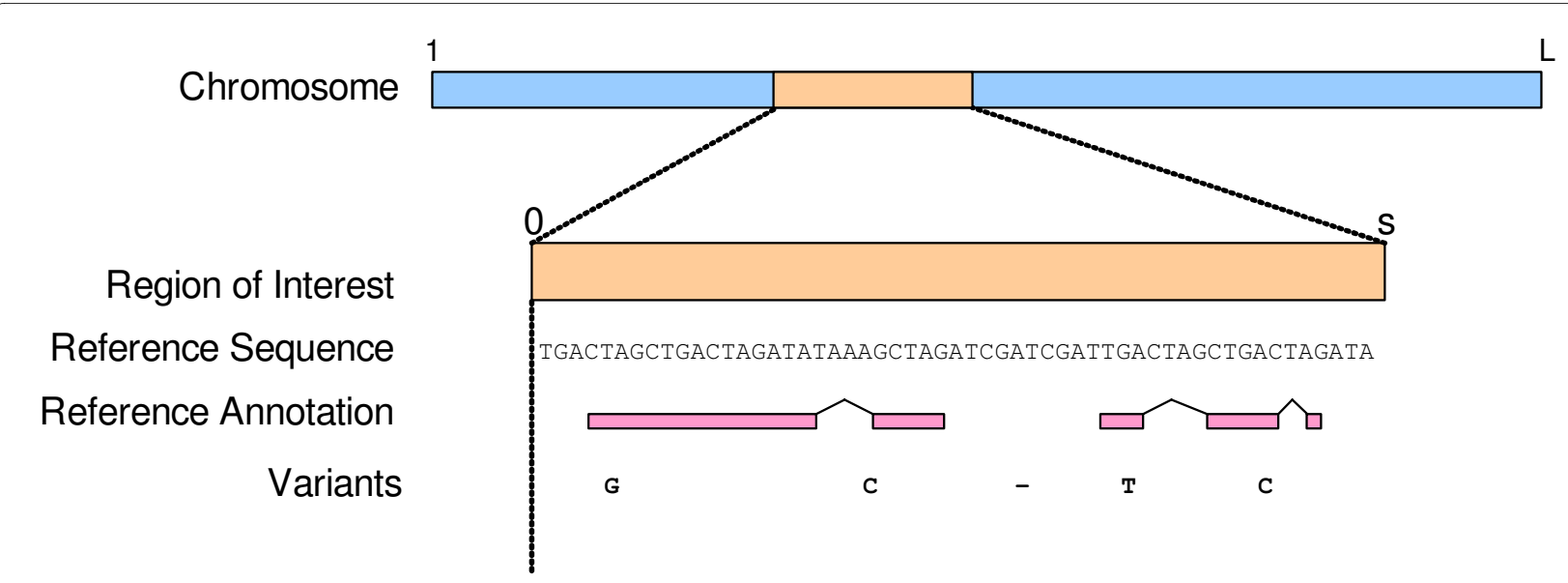

Figure 1 Relationship between query variants, reference annotation and sequence in the region of interest, and the complete reference genome/chromosome. For Ensembl genomes, the coordinate system is defined in 1-"residue"-based coordinates, from 1 to the length of the chromosome, $L$. The region of interest is a subset of the genome. The VariantClassifier uses a 0-"space"-based coordinate system to facilitate the description of indels. This local coordinate system ranges from 0 to the length of the reference sequence subregion, $s$. The reference annotation and query variants need to be specified in the region of interest's local coordinate system.

\section{1.) Reference nucleotide sequence FASTA file}

The reference sequence provides the coordinate system for the reference annotation and the query variants in the region of interest. The sequence is also utilized to make a protein sequence prediction, if a variant occurs in the coding sequence of the transcript. For large genomes, the reference nucleotide sequence will be a subregion of the complete genome.

\section{2.) Reference annotation file}

The reference annotation file, a tab-separated-value (TSV) text file, contains the positions of all the features annotated in the organism of interest in the local coordinates of the sequence file. For most investigators focused on a single organism or a single region of a genome, the annotation input will remain constant. For organisms, such as Homo sapiens, which has extensive annotation retrievable from Ensembl, a Perl application is included as part of the VariantClassifier package, which utilizes the Ensembl API to extract the annotation and nucleotide sequence file that the VariantClassifier application requires. For organisms without the benefit of the Ensembl resource, this annotation file will need to be generated using an alternative method, but only once.

\section{3.) Query variants file}

The query variants file is a TSV text file consisting of each variant's position on the reference nucleotide sequence, orientation, and assayed allele. Since the coordinates of the query variants file are in local 0 -space-based reference coordinates, the length of the allele on the reference is the distance between the specified begin and end coordinate. Therefore, if the mutant allele is the same length as the reference allele, a single nucleotide or block substitution is assumed. If the reference allele is shorter than the mutant allele, an insertion is assumed. A deletion is assumed if the reference allele is longer than the mutant allele. The information necessary to construct the query variants file is often available after reads have been mapped onto the reference genome. The format of this information will vary depending on the mapping software that has been chosen.

\section{Outputs}

There are two text file outputs from the VariantClassifier. The information contained between the two outputs are the same, but they are formatted differently:

\section{1.) Normalized output}

The normalized output should be read with an application that respects tab characters when displaying the text. The suggested viewer application could be a spreadsheet program such as OpenOffice Calc or Microsoft Excel. In this output format, information at the same classification hierarchy is displayed at the same tab stop position, or indentation distance.

\section{2.) Denormalized output}

The denormalized output was designed to be utilized by line-based parsers. Each line in this format contains every level of classification that could be assigned to each variant.

\section{Implementation}

For every variant, a series of assessments are made according to the decision tree shown in Figure 2. The decision tree is traversed from the root, located at the top of the graph, towards a leaf node, where a branch terminates. The decision to traverse a node, and receive a classification, is based on the variant's positional overlap with 


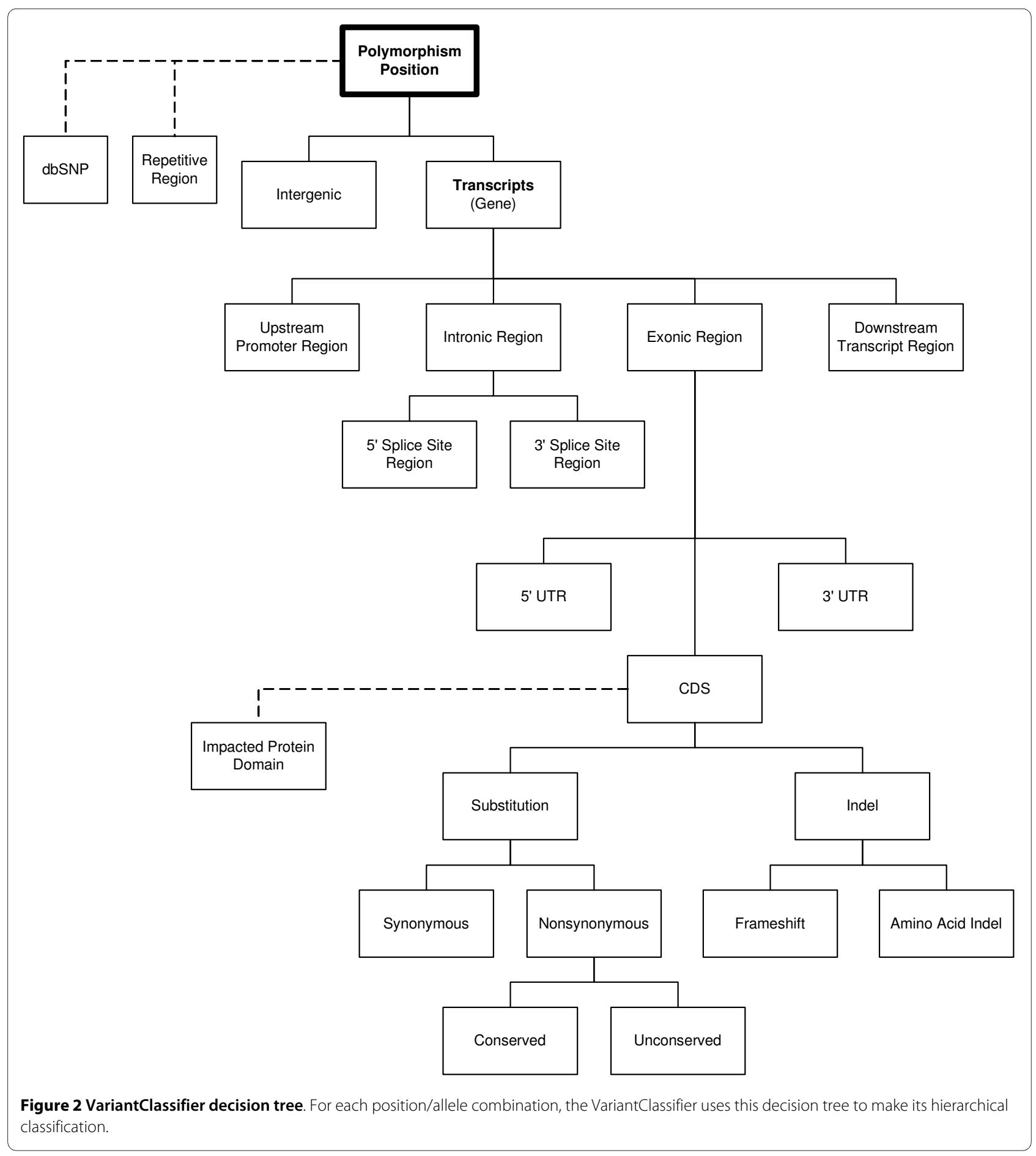

a feature's position that was described in the reference annotation file.

For every variant, an initial assessment of the variant's novelty or overlap with a repeat is generated if it collocates with an existing dbSNP ID or an annotated repeat, respectively. If the variant does not overlap with a gene, then it is considered intergenic. For each alternative transcript associated with a gene, if the variant falls in a region 1,000 bases upstream of the first exon or downstream of the last exon, it is considered in an "upstream promoter region" or "downstream transcript region", respectively. A distance to the nearest exon associated with the promoter region is provided. Variants found between exons are considered "intronic", and a " 5 ' Splice Site" or "3' Splice Site", is assigned if the variant is within 10 bases downstream or 6 bases upstream of an exon in 
its coding orientation. A distance to the nearest exon is provided. Variants considered "exonic" are further classified into "5' UTR", "CDS", or "3' UTR". Variants found in CDS have their positions mapped into amino acid coordinates, where they are assigned an "Impacted Protein Domain" based on Pfam annotation [5]. If the effect of the substitution is in a single codon, then the amino acid change is determined to be "synonymous" or "non-synonymous". Non-synonymous amino acid changes are classified as "Conserved" or "Unconserved." Indel variants are classified into "Frameshift" or "Amino Acid Indel", and a new protein sequence is predicted.

To determine whether variants causing non-synonymous substitutions are "conserved" or "unconserved", the BLOck SUbstitution Matrix (BLOSUM) [6] with a $30 \%$ cutoff is referenced. If the BLOSUM30 value for an amino acid transition is less than 0 , then the substitution is considered "unconserved". Any amino acid shift to a stop codon is also considered "unconserved". Because this BLOSUM-based assessment is quick and convenient, it is also relatively naïve and we recommend using SIFT [7] for a more thorough follow up assessment.

Additionally, flanking sequence surrounding the variant is also provided for future assay design, or for the requisite context needed for dbSNP submission.

\section{Conclusions}

To date, results from the VariantClassifier have contributed to the analyses of several large scale variant analyses publications $[8,9]$. The multi-level detailed nature of the output has made it possible to accurately assess the impact of novel variants quickly, effectively utilizing the annotation that is often available for organisms of interest that have been targeted for resequencing. The software is freely available on SourceForge.net.

\section{Availability and requirements \\ Project name: VariantClassifier \\ Project home page: http://www.jcvi.org/cms/research /projects/variantclassifier \\ Sourceforge Download: http://sourceforge.net/ projects/variantclass \\ Operating system: Tested and in production on Linux. Programming language: $\mathrm{Perl}$ \\ License: GNU GPL V3 \\ Any restrictions to use by non-academics: none}

Competing interests

The authors declare that they have no competing interests.

\section{Authors' contributions}

$\mathrm{KL}$ implemented the software. TBS and $\mathrm{KL}$ conceived of the study and validated the results. $\mathrm{KL}$ and TBS wrote the manuscript. All authors read and approved the final manuscript.

\section{Acknowledgements}

We acknowledge Samuel Levy, Jiaqi Huang, Ewen Kirkness, Qi Zhao, Anushka Brownley, Pauline Ng, Prateek Kumar and Vishal Thovarai for their valuable feedback. Their bug reports, additional feature requests, and dissemination of the tool, have made VariantClassifier robust and valuable to both internal and external collaborators. We would also like to thank Matthew LaPointe for developing the VariantClassifier website, which provides additional documentation and help information online.

\section{Author Details}

The J. Craig Venter Institute, 9704 Medical Center Drive, Rockville, MD 20850, USA

Received: 24 March 2010 Accepted: 13 July 2010

Published: 13 July 2010

\section{References}

1. Margulies M, Egholm M, et al:: Genome sequencing in microfabricated high-density picolitre reactors. Nature 2005, 437(7057):376-80.

2. Bentley DR, Balasubramanian S, Swerdlow HP, Smith GP, Milton J, Brown CG, Hall KP, Evers DJ, et al:: Accurate Whole Human Genome Sequencing using Reversible Terminator Chemistry. Nature 2008, 456(7218):53-59.

3. Sherry ST, Ward MH, Kholodov M, Baker J, Phan L, Smigielski EM, Sirotkin K. dbSNP: the NCBI database of genetic variation. Nucleic Acids Res 2001, 29(1):308-11.

4. Hubbard TJP, Aken BL, Beal K, Ballester B, Caccamo M, et al.: Ensembl 2007. Nucleic Acids Res 2007, 35:

5. Bateman A, Birney E, Cerruti L, Durbin R, Etwiller L, Eddy SR, Griffiths-Jones $\mathrm{S}$, Howe KL, Marshall M, Sonnhammer EL: The Pfam protein families database. Nucleic Acids Res 2002, 30(1):276-80.

6. Henikoff S, Henikoff JG: Amino acid substitution matrices from protein blocks. Proc Natl Acad Sci USA 1992, 89(22):10915-10919.

7. Ng PC, Henikoff S: Predicting deleterious amino acid substitutions. Genome Res 2000, 11(5):863-74.

8. Levy S, Sutton G, Ng PC, Feuk L, Halpern AL, et al:: The Diploid Genome Sequence of an Individual Human. PLoS Biol 2007, 5(10):

9. Ng PC, Levy S, Huang J, Stockwell TB, Walenz BP, Li K, Axelrod N, Busam DA, Strausberg RL, Venter JC: Genetic Variation in an Individual Human Exome. PLoS Genet 2008, 4(8):

doi: 10.1186/1756-0500-3-191

Cite this article as: Li and Stockwell, VariantClassifier: A hierarchical variant classifier for annotated genomes BMC Research Notes 2010, 3:191
Submit your next manuscript to BioMed Central and take full advantage of:

- Convenient online submission

- Thorough peer review

- No space constraints or color figure charges

- Immediate publication on acceptance

- Inclusion in PubMed, CAS, Scopus and Google Scholar

- Research which is freely available for redistribution
C Biomed Central 